\title{
Artigos Originais \\ Alterações Neurobiológicas Verificadas a partir do Tratamento com Terapia Cognitivo-comportamental no Transtorno Obsessivo-Compulsivo
}

\author{
Priscila Assumpção Fernandes \\ Marcele Regine de Carvalho \\ Universidade Federal do Rio de Janeiro
}

\begin{abstract}
RESUMO - Hipóteses neurobiológicas sobre o Transtorno Obsessivo-Compulsivo (TOC) sugerem alterações funcionais e anatômicas em determinadas áreas cerebrais relacionadas aos sintomas. Evidências indicam que a Terapia CognitivoComportamental (TCC) é eficaz para o tratamento do TOC e capaz de modular padrões neurais disfuncionais. O presente estudo objetivou descrever as alterações neurobiológicas promovidas pela TCC para o TOC. Realizou-se uma revisão sistemática, cuja amostra final correspondeu a cinco estudos. Em todos, houve melhora significativa do TOC e alterações neurobiológicas após a TCC, tais como redução da atividade e volume no córtex orbitofrontal, aumento da atividade no córtex cingulado anterior, tálamo e núcleo caudado. A TCC mostrou-se capaz de modular neurocircuitos envolvidos no TOC.
\end{abstract}

Palavras-chave: transtorno obsessivo-compulsivo, neurobiologia, terapia cognitivo-comportamental, revisão de literatura

\section{Neurobiological Changes after Cognitive-Behavioral Therapy of Obsessive- Compulsive Disorder}

\begin{abstract}
Neurobiological theories about Obsessive-Compulsive Disorder (OCD) signalize functional and anatomical changes in specific areas of the brain related to its symptoms. Evidence indicates that Cognitive-Behavioral Therapy (CBT) is effective in the treatment of OCD as well as capable of modulating dysfunctional neural patterns. This study aimed at describing neurobiological changes derived from the treatment of OCD with the CBT. A systematic review was carried out and its final sample consisted of five studies. In all of them, there was significant improvement of OCD as well as neurobiological changes, after CBT. The main findings were reduced activity and volume in the orbitofrontal cortex and increased activity in the anterior cingulate cortex, thalamus and caudate nucleus. CBT has shown to be able to modulate the neural circuits involved in OCD.
\end{abstract}

Keywords: obsessive-compulsive disorder, neurobiology, cognitive-behavioral therapy, literature review

O Transtorno Obsessivo Compulsivo (TOC) faz parte da categoria "Transtorno Obsessivo-Compulsivo e Transtornos Relacionados" da quinta edição do Manual Diagnóstico e Estatístico de Transtornos Mentais (DSM5; American Psychiatric Association [APA], 2013) e corresponde à presença de obsessões e/ou compulsões que se apresentam de forma a consumir tempo excessivo, causando prejuízos funcionais e sofrimento significativo. Obsessões são ideias, pensamentos, imagens ou impulsos vivenciados como intrusivos, apesar de serem claramente reconhecidos pelo indivíduo como algo vindo de si. Quando as obsessões aparecem, há um desconforto, sendo comum que o indivíduo tente ignorá-las, suprimi-las ou neutralizálas. Esta tentativa de alívio pode ocorrer através de uma compulsão. As compulsões são comportamentos repetitivos (por exemplo, realizar verificações) ou atos mentais (por exemplo, substituir pensamentos) aos quais o portador do TOC atribui a neutralização ou prevenção de um evento temido relacionado ao conteúdo da obsessão. Assim, obsessões levam a um aumento da ansiedade, cuja redução é associada às compulsões. Dessa forma, a pessoa pode passar a evitar gatilhos de obsessões e/ou dedicar elevada atenção às compulsões, processo que acarreta um impacto negativo em seu desempenho global (Cordioli, 2008).

1 Endereço para correspondência: Av. Dom Hélder Câmara, 5555 sala 412, Rio de Janeiro, RJ, Brasil. CEP. 20.770-145. E-mail: priscilafernandes.psi@gmail.com
Estudos epidemiológicos apontam que a prevalência do TOC na população geral é de 1,2\% em 12 meses e 2,3\% ao longo da vida (Goodwin, Beesdo-Baum, Knappe, \& Stein, 2013). A Organização Mundial da Saúde (OMS) o inclui entre as dez doenças com maior impacto em termos de incapacitação social no mundo, com tendência à cronicidade (Del-Porto, 2001). Diante do comprometimento funcional relacionado ao transtorno, é comum que os pacientes apresentem sintomas depressivos, que podem ser agravados por estratégias de enfrentamento inadequadas, que tendem a acentuar os sintomas obsessivo-compulsivos (Clark \& Beck, 2010a).

Um recente estudo de metanálise (Olatunji, Davis, Powers, \& Smits, 2013) sugere efeitos positivos decorrentes do uso da terapia cognitivo-comportamental (TCC) no tratamento do TOC. A TCC parte do princípio central de que as cognições (pensamentos) sobre a realidade (interna ou do ambiente) têm influência sobre as emoções e sobre os comportamentos, da mesma forma como o comportamento pode afetar os padrões de pensamento e as emoções (Pereira \& Rangé, 2011; Wright, Basco, \& Thase, 2008).

No modelo cognitivo do TOC, as obsessões derivam de pensamentos, imagens ou impulsos que aparecem de forma intrusiva sobre temas, em geral, indesejados. Porém, o fato de a maioria das pessoas experimentarem pensamentos indesejados, leva ao questionamento quanto ao processo pelo qual estes se tornariam obsessões (Rachman \& Silva, 
como citados por Cordioli \& Braga, 2011). Nesse processo, identifica-se o envolvimento de um processo metacognitivo, ou seja, se um pensamento intrusivo for considerado irrelevante, é mais provável que seja ignorado, mas, caso o sujeito o avalie como pessoalmente importante, inaceitável, imoral ou representante de uma ameaça, o mesmo poderá se tornar obsessivo. Assim, experimenta-se um desconforto, que poderá levar às compulsões, que incluem rituais, atos mentais e atividades encobertas. É comum que o indivíduo com TOC utilize estratégias para tentar interromper o pensamento, racionalizar, distrair-se, substituir pensamentos, promover autopunições, usar autorreasseguramento ou buscar reasseguramento de outros (Clark \& Beck, 2010a; Cordioli \& Braga, 2011). Porém, apesar de a compulsão levar a um alívio da ansiedade, este é momentâneo e reforçador do comportamento, visto que o faz parecer eficaz em atingir o objetivo (Cordioli \& Braga, 2011).

A estratégia de primeira escolha para o tratamento do TOC é a exposição e prevenção de respostas (EPR), que apresenta redução dos sintomas e manutenção dos ganhos. Nela há a exposição, ou seja, o contato direto e prolongado com a situação, objeto ou lugar evitado em função da ansiedade obsessiva, seguido da não realização do comportamento que aliviaria o desconforto (Cordioli, 2014). O fundamento da EPR é o fenômeno da habituação, ou seja, um mecanismo de aprendizagem que permite a adaptação a estímulos, inclusive aos que causariam desconforto. Dessa forma, quando não há um perigo real e a pessoa permanece em contato com o estímulo, a reação tende a ser reduzida (Cordioli, 2008). Cordioli e Braga (2011) defendem que, no momento inicial do tratamento, seja elaborada uma lista com os sintomas, que posteriormente será transformada em uma hierarquia, na qual os mesmos encontrem-se graduados de acordo com o desconforto causado, para serem enfrentados a partir do item mais fácil, em direção ao mais desconfortável.

O uso de intervenções cognitivas é necessário para que o paciente perceba o papel de suas avaliações sobre risco e vulnerabilidade e para que passe a interpretar as situações de forma mais acurada (Clark \& Beck, 2010b). O paciente deve aprender a diferenciar obsessões de suas avaliações sobre elas, considerando que a reestruturação cognitiva se direcionará para a última (Cordioli, 2008).

Quanto à neurobiologia do $\mathrm{TOC}$, uma hipótese atualmente aceita abrange uma disfunção no circuito orbitofrontalsubcortical (ou circuito cortico-estriado-tálamo-cortical), que conecta regiões do cérebro envolvidas no início de respostas comportamentais implementadas com pouco processamento consciente (Whiteside, Port, \& Abramowitz, 2004). Classicamente compreende-se esse circuito a partir de uma via direta e outra indireta. A via direta se projeta desde o córtex cerebral, conectando o estriado ao segmento interno do globo pálido e à parte reticulada da substância negra e para o tálamo, que projeta de volta para o córtex. Já a via indireta liga o estriado ao segmento externo do globo pálido e ao núcleo subtalâmico e então à parte reticulada da substância negra, ao tálamo e ao córtex. A atividade das vias direta e indireta deve promover o equilíbrio entre excitação e inibição do circuito descrito, possibilitando a modulação do movimento. O que se observa em pacientes com TOC é que algo rompeu o equilíbrio do circuito, tornando-o hiperativo (Cordioli, 2014;
Whiteside et al., 2004). As regiões do córtex orbitofrontal, núcleos da base e tálamo desempenham também um papel em respostas cognitivas (Lacerda, Dalgalarrondo, \& Camargo, 2001), visto que, quando a falha no processamento ocorre no circuito corticoestriatal pré-frontal, há o aumento da frequência de pensamentos obsessivos (Gomes, 2013)

A importância dos núcleos da base (que contemplam: núcleo estriado, formado pelo núcleo caudado e putâmen, globo pálido, substância negra e núcleo subtalâmico) foi destacada por Cordioli (2014), não só por seu papel na execução de atos motores, mas principalmente pela função na coordenação dos movimentos. Os núcleos da base relacionam-se com o TOC a partir do controle dos comportamentos, incluindo aprendizagem e reforçamento, bem como direcionamento cognitivo e motivacional.

A hipótese atualmente aceita é de que o equilíbrio entre as vias direta e indireta modula o tono inibitório proporcionado pelos núcleos da base para o tálamo. Assim, espera-se que sinais excitatórios (via direta) atinjam o estriado, aumentando os sinais inibitórios sobre células do globo pálido e substância negra, reduzindo a ação sobre o tálamo. O último, desinibido, facilitaria os movimentos e teria sua excitação em direção ao córtex aumentada (Cordioli, 2014). A atividade da via direta é contrabalanceada pela via indireta, que inibe o tálamo e o córtex. Sendo assim, o desequilíbrio dos tonos das vias direta e indireta provocariam a hiperatividade do circuito, que mediaria os comportamentos repetitivos. Defende-se também que os impulsos provenientes do tálamo atingiriam o córtex orbitofrontal, onde seria criado um reforço, mantendo o foco da atenção em preocupações obsessivas (Mercadante, Rosario-Campos, Quarantini, \& Sato, 2004).

Whiteside et al. (2004) observam que estudos com Tomografia por Emissão de Pósitrons (PET) têm mostrado um aumento no metabolismo cerebral da glicose em sujeitos com TOC no córtex orbitofrontal, núcleo caudado, tálamo, córtex pré-frontal e córtex cingulado anterior. Já em estudos cuja técnica utilizada foi a Tomografia por Emissão de Fóton Único (SPECT), foram encontrados tanto aumentos quanto diminuições do metabolismo em regiões que incluem córtex orbitofrontal, núcleo caudado, outras áreas corticais e o tálamo. Apesar das evidências, um estudo de metanálise (Whiteside et al., 2004) permitiu apontar que apenas alguns dados foram sustentados como sendo significativos. Em relação ao córtex orbitofrontal, as diferenças entre pacientes e controles mostraram-se significativas, sustentando a hipótese da hiperatividade. Já na área do núcleo caudado, estudos com PET indicam maior atividade, enquanto, em estudos com SPECT, foi encontrada atividade reduzida (Whiteside et al., 2004). Os autores (Whiteside et al., 2004) destacam ainda que os achados em direções opostas encontrados em estudos utilizando PET e SPECT não representam contraditoriedade, mas expressam os diferentes métodos envolvidos, pois enquanto uma região pode ter aumentado seu metabolismo por um aumento da ativação, pode também ter o fluxo sanguíneo reduzido por uma constrição dos vasos sanguíneos. Além disso, o pareamento de aumento do metabolismo com a redução do fluxo sanguíneo é algo pouco comum e que, caso seja replicado, pode indicar um marcador de neuroimagem para o TOC. Outra interpretação para a aparente contraditoriedade dos achados corresponde à 
possibilidade de alterações metabólicas do TOC levarem ao esmaecimento da substância utilizada no SPECT, podendo assim não refletir uma redução real do fluxo sanguíneo cerebral regional, produzindo as discrepâncias (Whiteside et al., 2004).

Já em relação ao volume, a metanálise conduzida por Peng et al. (2012) indica uma redução significativa da substância cinzenta no córtex orbitofrontal, córtex cingulado anterior, assim como em regiões dorsolaterais do córtex pré-frontal. A última apresenta relação com os prejuízos cognitivos observados no TOC, como dificuldades no planejamento, tomada de decisão e aprendizagem reversa.

Volumes aumentados de substância cinzenta foram observados no núcleo lentiforme direito (que inclui o putâmen), estendendo-se até o núcleo caudado. Essa evidência corrobora a hipótese do envolvimento dos gânglios basais no TOC, que desempenham um papel significativo na previsão de eventos futuros, reforçando comportamentos desejados e suprimindo os não desejados. A disfunção apresentada é compatível com a dificuldade na supressão de comportamentos no TOC (Peng et al., 2012). Nesse estudo, foi encontrado também um aumento no volume de substância cinzenta na região superior direita do córtex parietal, cuja função inclui o deslocamento da atenção e inibição de resposta, deficitários no transtorno citado. As evidências encontradas apontam para o envolvimento do circuito cortico-estriado-talâmico no TOC, responsável por funções motoras e executivas (Peng et al., 2012). Por fim, apesar da tendência indicada pelos dados descritos, estudos sobre a fisiopatologia do TOC ainda podem ser considerados inconclusivos (Haber \& Heilbronner, 2013).

A investigação neurobiológica torna-se também relevante diante das evidências clínicas e experimentais obtidas em estudos realizados a partir de técnicas de neuroimagem funcional, cujos resultados apontam para alterações neurais a partir de intervenções psicoterapêuticas. Alguns estudos demonstram alterações cerebrais funcionais após o tratamento com TCC (para uma revisão, ver Clark \& Beck, 2010c). Além disso, foi verificado que o padrão de comunicação sináptica pode ser alterado de forma análoga à ação de psicofármacos (Landeira-Fernandez, 2011). O objetivo principal do presente estudo é realizar uma revisão sistemática da literatura, com a finalidade de descrever as alterações neurobiológicas verificadas através de técnicas de neuroimagem após o tratamento do TOC com TCC em adultos.

\section{Método}

As buscas foram conduzidas em 8 de junho de 2013 nas bases de dados eletrônicas ISI Web of Science e PubMed. Não foram utilizados filtros para a pesquisa. Em ambas as bases, foi utilizada a opção Advanced Search, sendo os seguintes termos buscados e, em seguida, combinados pelo operador $A N D$ :

\footnotetext{
- CBT OR "cognitive *therap*" OR "cognitive behav**therap*" OR "behav**therap*"

- Neuroimag* OR imag*

- "Obsessive compulsive disorder"
}

A escolha dos termos referentes à abordagem psicoterápica pesquisada buscou abranger estudos com enfoque comportamental e cognitivo-comportamental. Na revisão sistemática, foram incluídos artigos experimentais e em inglês, que descreveram estudos clínicos com pacientes adultos diagnosticados com TOC, nos quais se realizou avaliação utilizando neuroimagem, nos momentos pré e pós-tratamento psicoterápico. As técnicas de neuroimagem incluídas foram:

- Ressonância Magnética Estrutural (RM);

- Ressonância Magnética Funcional (RMF);

- $\quad$ SPECT;

- PET.

Foram encontrados 368 estudos e, após a exclusão das repetições, 12 preencheram os critérios para inclusão. Após, foram excluídos mais sete estudos, sendo dois por contarem com caso único e cinco por utilizarem como referência diagnóstica a terceira edição do DSM Portanto, o número final de estudos analisados foi cinco. É importante ressaltar que entre esses, um não deixou clara a referência para o diagnóstico do TOC, porém a partir do texto inferiu-se que os critérios eram compatíveis com os contidos na quarta edição do DSM.

\section{Resultados}

Os cinco estudos foram analisados e a síntese dos dados relativos às amostras dos estudos e aos métodos utilizados encontram-se, respectivamente, nas Tabelas 1 e 2.

Hoexter et al. (2011) realizaram um estudo experimental randomizado e controlado com o objetivo de, a partir de imagens obtidas por RM, documentar o perfil estrutural das anormalidades associadas ao TOC em pacientes sem tratamento psicoterápico ou farmacológico prévio, comparando pacientes tratados com TCC ou fluoxetina, com controles saudáveis. $\mathrm{O}$ tratamento em TCC foi realizado em grupos compostos por 6 a 8 pessoas, em sessões semanais com duração de 2 horas, por 12 semanas, a partir do protocolo publicado por Cordioli e colaboradores (como citados por Hoexter et al., 2011). As sessões consistiram em psicoeducação, construção de hierarquia para a realização de EPR, técnicas cognitivas, revisão do tratamento, além de sessão com familiares. No protocolo utilizado, a EPR tem um grande destaque, sendo realizada ao longo da maior parte do tratamento. Foram realizadas avaliações para apurar a modulação do tratamento, antes e após o mesmo. Durante as sessões de aquisição de imagens com RM, o foco foi direcionado para áreas previamente identificadas como sendo implicadas no TOC: córtex orbitofrontal, córtex cingulado anterior, corpo estriado, tálamo e estruturas temporo-límbicas.

Ao comparar as imagens de pacientes e controles, obtidas no pré-tratamento, foram encontrados volumes menores de substância cinzenta nas áreas do putâmen esquerdo (porções dorsais rostrais), diferença que se manteve ao serem retirados da análise os pacientes portadores de transtorno depressivo maior. Entretanto, esse grupo de pacientes não deprimidos $(n=13)$ apresentou volumes menores de SC bilateralmente no córtex orbitofrontal medial e córtex cingulado anterior direito. 
Os resultados do exame após o tratamento não identificaram diferenças significativas em relação ao volume de substância cinzenta entre pacientes e controles, indicando que houve a modulação a partir da TCC. Os autores concluíram que as diferenças encontradas entre pacientes e controles somente antes do tratamento reforçam o envolvimento do córtex orbitofrontal medial e córtex cingulado anterior direito na fisiopatologia do TOC.

Outro estudo, conduzido por Apostolova et al. (2010), buscou investigar os efeitos do tratamento com farmacoterapia e TCC no metabolismo da glicose cerebral, além de testar se esses processos no córtex orbitofrontal ou no núcleo caudado são indicadores do nível de resposta ao tratamento. O tratamento com TCC foi realizado individualmente, a partir de um modelo multimodal de tratamento (Hand, como citado por Apostolova et al., 2010), que consistia em 40 horas de tratamento distribuídas de acordo com cada caso, variando entre sete e 20 semanas. As estratégias trabalhadas envolviam análise dos elementos dos sintomas do TOC, intervenções cognitivas e exposição in vivo, que é a modalidade de exposição em que se entra em contato real com o elemento ansiogênico (Cordioli, 2014), com prevenção de respostas. A avaliação neurobiológica enfatizou bilateralmente o Córtex Orbitofrontal e o núcleo caudado para a realização de PET com 18F-Fluorodeoxiglicose (18F-FDG), antes e após o tratamento. Foram considerados responsivos ao tratamento os participantes cujo escore do Y-BOCS apresentasse redução superior a $35 \%$ para a soma de compulsões e obsessões. O Y-BOCS consiste em uma escala formato Likert, a partir da qual são gerados escores individuais para obsessões e compulsões, além da soma das duas, indicando nível de severidade do transtorno (Goodman et al., 1989) Entre os pacientes que responderam ao tratamento, houve um aumento significativo da taxa metabólica da glicose local (SLMRG1c) no núcleo caudado direito, que se correlacionou positivamente com a melhora no escore do Y-BOCS. Os autores destacam que não houve diferença significativa encontrada após o tratamento no córtex orbitofrontal, apesar de se existir uma tendência a uma correlação negativa entre a porcentagem de mudança no escore do Y-BOCS e o SLMRG1c após o tratamento no córtex orbitofrontal. Diante dos resultados, os autores concluíram que o aumento de SLMRG1c no núcleo caudado direito foi um resultado do sucesso das terapias. O que permanece obscuro é se esse

Tabela 1. Características das amostras dos estudos selecionados

\begin{tabular}{|c|c|c|c|c|c|c|c|}
\hline Referência & Sujeitos(n) & Gênero & Idade M(DP) & Características & Comorbidades (n) & Critérios de exclusão & Medicação \\
\hline Hoexter et al, 2011 & $\begin{array}{l}26 \mathrm{P}(13 \text { para } \\
\mathrm{TCC}) / 36 \mathrm{C}\end{array}$ & $23 \% \mathrm{~F}$ & $31,5( \pm 10,2)$ & $\begin{array}{l}\text { Escorte total do Y-BOCS } \geq 16 \\
\text { para compulsões ou obsessões; } \\
\text { Ou } \geq 10 \text { considerando para } \\
\text { compulsões ou obsessões. }\end{array}$ & $\begin{array}{l}\text { TDM atual(13), histórico } \\
\text { TDM ou Dis (10), Ago } \\
\text { (10), TPA (1), TP (3), } \\
\text { Fesp (13), Fsoc (21), } \\
\text { TEPT (8), TAG (17), } \\
\text { TBip I(1), TBip II } \\
\text { (2), somatização (2), } \\
\text { hipocondria (1), TDM } \\
\text { (5), AN (3), BN (2), } \\
\text { CA (2), skin picking } \\
\text { (8), TTM (2), TEI (7), } \\
\text { TDA/H (3), tics cronicos } \\
\text { (4), TT (2). }\end{array}$ & $\begin{array}{l}\text { Qualquer exposição a } \\
\text { psicotrópicos ou a pelo } \\
\text { menos } 12 \text { sessões de TCC, } \\
\text { ferimento na cabeça com } \\
\text { perda de consciência, } \\
\text { abuso ou dependência de } \\
\text { substâncias, psicose, risco } \\
\text { de suicídio, doença que } \\
\text { afete o sistema nervoso, } \\
\text { contra indicações para MRI, } \\
\text { gravidez. Idade menor que } 18 \\
\text { anos e maior que } 65\end{array}$ & Nenhuma \\
\hline Apostolova etal, 2010 & $16 \mathrm{P}(9 \mathrm{TCC})$ & $4 \mathrm{~F} / \% \mathrm{M}$ & $37,1( \pm 10,3)$ & $\begin{array}{l}\text { Diagnóstico de TOC por pelo } \\
\text { menos } 2 \text { anos. }\end{array}$ & $\begin{array}{l}\text { TDM (1), TPA (1), TP } \\
\text { (1), Dis (3) }\end{array}$ & $\begin{array}{l}\text { Idade menor que } 18 \text { anos, } \\
\text { gravidez, doença fisica } \\
\text { aparente, histórico de } \\
\text { doenças neurológicas ou } \\
\text { de abuso de substâncias, } \\
\text { sintomas motores } \\
\text { perceptíveis, sintomas } \\
\text { psicóticos. }\end{array}$ & $\begin{array}{l}\text { Nenhuma, pelo menos } \\
3 \text { semanas antes da } \\
\text { inclusão. }\end{array}$ \\
\hline Freyer et al, 2011 & 10 P $10 \mathrm{C}$ & $3 \mathrm{~F}$ & $36,1( \pm 9,36)$ & Pacientes internados & Histórico bulimia (1) & $\begin{array}{l}\text { Prejuízo neurolótico, } \\
\text { ferimento na cabeça, abuso } \\
\text { de substâncias, episódios } \\
\text { psicóticos atuais ou passados, } \\
\text { gravidez e idade maior } \\
\text { que } 65 \text { anos e uso atual de } \\
\text { psicotrópricos }\end{array}$ & $\begin{array}{l}\text { Nenhuma, pelo menos } \\
4 \text { semanas antes da } \\
\text { inclusão }\end{array}$ \\
\hline Yamanishi et al, 2009 & $\begin{array}{l}45(33 \text { Resp; } \\
12 \text { N-resp) }\end{array}$ & $26 \mathrm{~F} / 19 \mathrm{M}$ & $\begin{array}{l}\text { Resp: } 34,7( \pm 7,1) / \\
\text { N-resp: } 32,1( \pm 7,1)\end{array}$ & $\begin{array}{l}\text { Não responsivos à } \\
\text { farmamacoterapia }\end{array}$ & Sem comorbidades & $\begin{array}{l}\text { Uso de outro psicotrópico, } \\
\text { além de SRI, doenças } \\
\text { neurológicas atuais ou } \\
\text { passadas ou outras doenças } \\
\text { significativas, dependência de } \\
\text { substâncias, retardo mental, } \\
\text { gravidez, outro transtorno } \\
\text { eixo I. }\end{array}$ & SRIs \\
\hline Saxena et al, 2008 & $10 \mathrm{P} 12 \mathrm{C}$ & $6 \mathrm{~F} / 4 \mathrm{M}$ & $40,6( \pm 12,3)$ & - & TDM (1) & $\begin{array}{l}\text { Condições médicas } \\
\text { significativas, abuso de } \\
\text { substâncias autal ou recente, } \\
\text { outro transtorno eixo I } \\
\text { (Exceto um paciente com } \\
\text { depressão). }\end{array}$ & $\begin{array}{l}\text { Seis pacientes } \\
\text { medicados: SRIs } \\
\text { (6), Buspirona (3), } \\
\text { Risperidona (3) e } \\
\text { Clonazepam (2). Sem } \\
\text { alteração da dose } \\
\text { por pelo menos } 12 \\
\text { semanas antes do } \\
\text { início. }\end{array}$ \\
\hline
\end{tabular}

Nota: P: pacientes; C: controle; M: Sujeitos do sexo masculino; F: Sujeitos do sexo feminino; M: média; DP: Desvio Padrão; Resp: Responsivos; N-resp: não responsivos; TDM: Transtorno depressivo maior; TPA Transtorno de pânico com agorafobia, TP : Transtorno de pânico sem agorafobia; Dis: Sistimia; SRIs: Inibidor de recaptação de serotonina, Ago: Agorafobia, Fesp: Fobia específicia. Fsoc: Fobia social, TEPT: Transtorno de estresse pós-traumático, TAG: Transtorno de ansiedade generalizada, TBip: Transtono bipolar, TDM: Transtorno dismórfico corporal, AN: Anorexia nervosa; BN: Bulimia nervosa ,CA: Compulsão alimentar, TTM: Tricotilomania, TEI: Transtorno explosivo intermitente, TDA/H: Transtorno do déficit de atenção/hiepratividade, TT: Tourette. 
Tabela 2. Descrição do método dos estudos selecionados

\begin{tabular}{|c|c|c|c|c|c|c|c|}
\hline Referência & Delineamento & Estímulo & Escalas Utilizadas & Tipo Scanner & $\begin{array}{l}\text { Escalas pré- } \\
\text { tratamento }\end{array}$ & $\begin{array}{l}\text { Imagens pré- } \\
\text { tratamento }\end{array}$ & Resultados \\
\hline Hoexter et al, 2011 & $\begin{array}{l}\text { Pacientes avaliados antes } \\
\text { e após o tratamento } \\
\text { oferecido com TCC ou } \\
\text { fluoxetina, separados de } \\
\text { forma randomizada, e } \\
\text { comparados com grupo de } \\
\text { controle }\end{array}$ & Repouso & $\begin{array}{l}\text { Y-BOCS, D-YBOSC, } \\
\text { BDI, BAI, CGI }\end{array}$ & $\operatorname{MRI}(1,5 \mathrm{~T})$ & $\begin{array}{c}\text { Y-BOCS: } 25,1 \\
( \pm 5,2) ; \text { BDI: } \\
17,3( \pm 9,4)\end{array}$ & $\begin{array}{l}\text { Menor volume } \\
\text { SC putâmen } \\
\text { esquerdo, COF } \\
\text { medial CCA } \\
\text { direito }\end{array}$ & $\begin{array}{l}\text { Redução do volume de } \mathrm{SC} \text { no pós tratamento } \\
\text { no putamen esquerdo }(0,2 \%) \text {, putamen } \\
\text { direito }(1,4 \%) \text { e COF medial esquerdo. } \\
\text { Aumento volumento de SC no CCA esquerdo } \\
(2,8 \%) \text {. Redução de } 33 \% \text { do Y-BOCS e } \\
38,1 \% \text { BDI. }\end{array}$ \\
\hline Apostolova etal, 2010 & $\begin{array}{l}\text { Pacientes escolheram } \\
\text { entre tratamento com } \\
\text { partoxetina ou TCC. Os } \\
\text { grupos foram avaliados } \\
\text { antes e após o tratamento. }\end{array}$ & $\begin{array}{l}\text { Repouso, cabeça } \\
\text { imobilizada. }\end{array}$ & $\begin{array}{l}\text { Y-BOCS, HAMD, } \\
\text { BDI, }\end{array}$ & $\begin{array}{l}\text { FDG PET } \\
\text { (ECAT } \\
\text { EXACT } \\
921 / 47)\end{array}$ & $\begin{array}{c}\text { Y-BOCS: } 23,2 \\
( \pm 8,1) ; \text { BDI } \\
12,9( \pm 8,2)\end{array}$ & $\begin{array}{l}\text { Não } \\
\text { disponíveis }\end{array}$ & $\begin{array}{l}\text { REdução Y-BOCS } 47,1( \pm 27,1) \% \text { e BDI } 48 \\
( \pm 51) \%{ }^{*} \text {. Aumento significativo de } 0,13 \text { da } \\
\text { taxa metabólica da glicose local no núcleo } \\
\text { caudado direito entre Resp. }\end{array}$ \\
\hline Freyer et al, 2011 & $\begin{array}{l}\text { Pacientes internados } \\
\text { foram tratados com TCC } \\
\text { e, após a avaliação antes } \\
\text { e após e comparados com } \\
\text { grupo controle. }\end{array}$ & $\begin{array}{l}\text { Tarefa de } \\
\text { aprendizagem } \\
\text { reversa } \\
\text { envolvendo erro } \\
\text { probabilístico. }\end{array}$ & HAMD, Y-BOCS & $\begin{array}{l}\text { fMRI (Trio } \\
3 \mathrm{~T})\end{array}$ & $\begin{array}{l}\text { Y-BOCS: } 25,4 \\
\quad( \pm 4,81)\end{array}$ & $\begin{array}{l}\text { Reduzida } \\
\text { ativação } \\
\text { no núcleo } \\
\text { caudado } \\
\text { direito, COF } \\
\text { bilateral e } \\
\text { putamen } \\
\text { direito }\end{array}$ & $\begin{array}{l}\text { Redução Y-BOCS } 14,2 \text { (Fraco); Ativação } \\
\text { no COF direito manteve-se reduzida e foi } \\
\text { normalizada no COF esquerdo e putâmen; } \\
\text { Ativações diferenciais no núcleo caudado. }\end{array}$ \\
\hline Yamanishi et al, 2009 & $\begin{array}{l}\text { Após tratamento com } \\
\text { terapia comportamental, } \\
\text { apcientes foram separados } \\
\text { em dois grupos: não- } \\
\text { responsivos, caso Y-BOCS } \\
\text { reduzisse menos de } 40 \% \text {, } \\
\text { e responsivos. }\end{array}$ & $\begin{array}{l}\text { Repousos, sem } \\
\text { falar e imóveis. }\end{array}$ & $\begin{array}{l}\text { Y-BOCS, BDI-II, } \\
\text { STAI (S e T) }\end{array}$ & $\begin{array}{l}\text { SPECT } \\
\text { (ECAM)/ } \\
(1,5 \mathrm{~T})\end{array}$ & $\begin{array}{c}\text { Y-BOCS: } \\
\text { Resp: } 33,6 \\
( \pm 4,5) / \\
\text { n-Resp: } \\
34,4 \text { ( } \pm 4,8) ; \\
\text { BDI-II Resp: } \\
\text { 15,9( } \pm 6,7) / \\
\text { n-Resp:16,1 } \\
( \pm 7,3)\end{array}$ & $\begin{array}{l}\text { Não } \\
\text { disponíveis }\end{array}$ & $\begin{array}{l}\text { Redução Y-BOCS de } 51,4( \pm 7,1) \% \text { em Res; } \\
\text { e 13,7 ( } \pm 7,3) \% \text { em n-Resp; BDI-II Resp: } \\
\text { 15,4( } \pm 6,1) \text {, n-Resp: } 15,1( \pm 6,9) \text {. REdução } \\
\text { FSCr no COF direito, CPF medial direito, } \\
\text { giero frontal médio, córtex frontal inferior } \\
\text { direito; Aumento FSCr no giro fusiforme } \\
\text { direito, cuneos direito, giro angular direito. }\end{array}$ \\
\hline Saxena et al, 2008 & $\begin{array}{l}\text { Pacientes foram } \\
\text { comparados a controles } \\
\text { suadáveis. }\end{array}$ & $\begin{array}{l}\text { Repouso, } \\
\text { sem falar e } \\
\text { imóveis. Cabeça } \\
\text { imobilizada. }\end{array}$ & $\begin{array}{l}\text { Y-BOCS, HAMD, } \\
\text { HAS, GAS, CGI }\end{array}$ & $\begin{array}{l}\text { PET }(\text { CTI } \\
\text { EXACT } \\
\text { HR1961)/ } \\
\text { MRI }(1,5 T)\end{array}$ & $\begin{array}{l}\text { Y-BOCS: } \\
25,1( \pm 3,3)\end{array}$ & $\begin{array}{l}\text { Não } \\
\text { disponíveis }\end{array}$ & $\begin{array}{l}\text { Y-BOCS: } 11,05(5,1) \text { (fraco). } 9 \text { pacientes } \\
\text { considerados responsivos (queda de no } \\
\text { mínimo } 35 \% \text { do Y-BOCS). Aumento do } \\
\text { metabolismo cerebral da glicose no CCA } \\
\text { dorsal direito e redução em tálamo bilateral. }\end{array}$ \\
\hline
\end{tabular}

Nota: BDI: Beck Depression Inventory; BAI: Beck Anxiety Inventory; CGI: Clinical Global Impression; STAI: State-Trait Anxiety Inventory; HAS: Hamilton Anxiety Scale; GAS: Global Assessment Scale

efeito é primário, relacionado à melhora dos sintomas do TOC, ou se aparece em decorrência da melhora nos sintomas depressivos. Sendo assim, o sofrimento relacionado ao TOC deve ser considerado uma importante covariável na avaliação dos efeitos da terapia no metabolismo de glicose. Tal sugestão vale tanto para sujeitos que preenchem critério para o transtorno depressivo maior associado ao TOC, quanto para os que não preenchem.

Outro estudo, publicado por Freyer et al. (2011), buscou investigar o impacto da psicoterapia na capacidade de resposta frontoestriatal, utilizando como parâmetro um teste de flexibilidade cognitiva. Era esperado que, antes do tratamento, os pacientes apresentassem uma reduzida ativação nesse circuito, que se normalizaria após a TCC. A amostra contou com pacientes internados no Hospital Universitário de Freiburg, além do grupo controle de sujeitos saudáveis. $\mathrm{O}$ tratamento psicoterápico consistiu em sessões individuais de TCC com duração entre 8 e 12 semanas, seguindo o protocolo de Hohagen (como citado por Freyer et al., 2011). A primeira parte somou 6 horas de avaliação e planejamento do tratamento, durante as quais eram fornecidas informações sobre o modelo do TOC e sobre a TCC. Já a segunda parte durou entre quatro e seis semanas, contando com 16 sessões que se estenderam entre 90 e 120 minutos de EPR. As exposições foram realizadas juntamente com o terapeuta, que também prescrevia que os pacientes as continuassem entre as sessões. É importante destacar que as sessões de EPR eram in vivo e incluíam sessões em casa. Durante o escaneamento realizado a partir da RMF, foi realizada uma tarefa de aprendizagem de reversão probabilística, que buscou sondar capacidades adaptativas de alternar estratégias. Após um breve treino sem envolver reversão, os sujeitos iniciavam a tarefa, durante a qual deveriam escolher um padrão correto entre duas opções, apertando um botão direito ou esquerdo. O feedback da resposta era dado por um pictograma de um rosto feliz ou triste, apresentado imediatamente após cada escolha. Após rodadas de 10 a 15 (randomizado) respostas corretas, os sujeitos precisavam adaptar a estratégia anterior, selecionando o estímulo previamente incorreto. Adicionalmente, erros probabilísticos eram intercalados, levando a um feedback negativo diante de uma resposta correta. Deveriam responder o mais rápido o possível, mas sem comprometer a precisão. Caso o sujeito alternasse a estratégia após o erro probabilístico, era considerado erro na pontuação final. Durante o escaneamento, foram executadas duas sessões sucessivas, cada uma com duração de nove minutos e com 10 regras apresentadas (nove etapas de reversão). Cada etapa continha entre 0 e 4 erros probabilísticos de feedback. Ao final, os erros espontâneos eram calculados de forma separada às mudanças de estratégia após o erro probabilístico.

Antes do tratamento, foi possível identificar uma correlação negativa tanto entre o escore do Y-BOCS e o número de erros espontâneos, quanto entre o escore do Y-BOCS e o número de erros provenientes da mudança desnecessária da estratégia após o erro do feedback. Tais correlações não apareceram após o tratamento. Nas primeiras avaliações, também foram encontrados níveis menores de ativação em pacientes, em comparação aos controles, bilateralmente no córtex orbitofrontal e no putâmen. Após o tratamento, ainda havia uma diferença, porém somente no córtex orbitofrontal direito. Pacientes com reduções 
mais significativas do Y-BOCS tiveram menor aumento na ativação do globo pálido. $\mathrm{O}$ estudo, ainda que preliminar, apresenta evidências de mudanças neuronais após a TCC nos núcleos da base como parte da rede frontoestriatal implicada no TOC. Os autores sugerem que a TCC parece agir, em geral, desde o córtex pré-frontal dorsolateral dominante para alvos cognitivos mais distantes, como o córtex orbitofrontal e os núcleos da base.

Yamanishi et al. (2009) selecionaram pacientes considerados não responsivos ao tratamento (duração mínima de 3 meses) com Inibidores Seletivos de Recaptação de Serotonina (ISRS) para estudar as mudanças no Fluxo Sanguíneo Cerebral Regional (FSCr) após a Terapia Comportamental (TC). O objetivo da pesquisa foi avaliar o FSCr entre os pacientes considerados, após o tratamento psicoterápico, responsivos (redução de pelo menos $40 \%$ na pontuação do Y-BOCS) e os não-responsivos. Outro objetivo do estudo foi determinar se o FSCr pré-tratamento seria preditivo de resposta. O tratamento foi realizado a partir do protocolo de Likura (como citado por Yamanishi et al., 2009), contendo sessões individuais de TC com duração de 12 semanas. Os encontros tinham duração de 45 minutos e eram realizados entre 1 e 5 vezes por semana. A $1^{\mathrm{a}}$ sessão objetivou psicoeducar sobre o TOC e sobre o modelo comportamental do tratamento. O objetivo da $2^{\mathrm{a}}$ sessão foi planejar o tratamento, baseando-se na análise comportamental e na hierarquia gradual para exposição. Após, eram iniciadas as sessões de EPR. Além das sessões semanais, os pacientes recebiam como tarefa de casa a realização de exposições por aproximadamente 3 horas, 5 vezes por semana. Nas últimas sessões, eles deveriam realizar os itens de maior dificuldade da hierarquia. Inicialmente, a amostra era homogênea, ou seja, não havia diferenças significativas nas diferentes medidas entre os pacientes que seriam posteriormente classificados como responsivos e não responsivos. Considerando os pacientes responsivos, foi encontrada correlação negativa entre Y-BOCS e FSCr pré-tratamento no córtex orbitofrontal bilateral (ou seja, quanto maior o FSCr pré-tratamento, maior a redução na pontuação do Y-BOCS). Porém, ao considerar toda a amostra, foi possível notar uma correlação negativa entre o Y-BOCS e o FSCr pré-tratamento no córtex orbitofrontal direito. Após o tratamento, o FSCr foi menor entre os considerados responsivos, quando comparados aos não responsivos, nas áreas do giro frontal médio bilateral e córtex pré-frontal medial direito. Entre os responsivos, houve reduções no FSCr, ao comparar valores pré e pós-tratamento, nas áreas do giro frontal médio, córtex pré-frontal medial direito, córtex frontal inferior direito e córtex orbitofrontal direito. Por outro lado, houve um aumento do FSCr no giro fusiforme direito, cuneus direito (no córtex occipital) e giro angular direito (no córtex parietal). Já entre os não-responsivos, não foram encontradas diferenças antes e após o tratamento.

Com os achados, os autores concluíram que maiores níveis do FSCr no córtex orbitofrontal bilateral poderiam ser preditivos de uma melhor resposta ao tratamento com TC. O estudo sugere que, além do córtex orbitofrontal, há também ligação do TOC com as áreas do giro frontal médio esquerdo, córtex pré-frontal medial direito e córtex frontal inferior direito. $\mathrm{O}$ estudo suporta a modulação da função neural no córtex orbitofrontal e no córtex frontal medial em resposta à $\mathrm{TC}$.

Saxena et al. (2009) desenvolveram um estudo cujo objetivo foi esclarecer a mediação cerebral da resposta ao tratamento breve e intensivo com TCC para o TOC. Outro alvo foi determinar se a TCC administrada de forma intensiva seria capaz de induzir rapidamente a mudanças vistas após farmacoterapia e TCC padrão (com frequência semanal de sessões). O tratamento seguiu o protocolo de Foa et al. (como citados por Saxena et al., 2009) e consistiu em sessões de TCC com duração de 90 minutos, com frequência de 5 vezes por semana, durante 4 semanas. As sessões envolviam EPR e, além delas, o paciente deveria realizar 4 horas de tarefas de casa, bem como técnicas cognitivas e de mindful awareness (técnicas que envolvem atenção plena, foco no presente e aceitação dos estados emocionais sem julgamento). A exposição foi graduada com situações reais e imaginárias. Da primeira até a terceira sessão, o objetivo foi realizar avaliação comportamental, treino em automonitoria e discussão sobre os objetivos específicos do paciente, além da elaboração da hierarquia para exposição. A partir da $4^{\mathrm{a}}$ até a $15^{\mathrm{a}}$ sessão, foram realizadas exposições imaginárias e in vivo, não só nas sessões, mas também como tarefa de casa. Entre a $16^{\mathrm{a}}$ e a $20^{\mathrm{a}}$ sessões, o trabalho voltou-se para a prevenção de recaídas e continuação da EPR, além de reestruturação cognitiva e avaliação do progresso. Os pacientes deveriam aprender a reconhecer os sinais internos e externos que funcionavam como gatilho para os sintomas do TOC (mindful awareness). Os efeitos da TCC resultaram em significativas mudanças no metabolismo cerebral da glicose: aumento significativo na atividade no córtex cingulado anterior dorsal direito, correlacionando-se negativamente com o escore do Y-BOCS, além de declínio na atividade bilateral talâmica.

\section{Discussão}

O presente estudo revisou de forma sistemática a literatura acerca das alterações neurobiológicas decorrentes de intervenções cognitivo-comportamentais no TOC. Entre os estudos analisados, todos apresentaram uma redução significativa nos escores da escala Y-BOCS após o tratamento, realizado a partir de protocolos de TCC já validados, o que corrobora a eficácia das intervenções.

As pesquisas que apresentaram dados antes do tratamento apontaram volumes menores de substância cinzenta no córtex orbitofrontal e córtex cingulado anterior (Hoexter et al., 2011) além de menor ativação no núcleo caudado (Freyer et al., 2011), mostrando relação com os dados descritos sobre bases neurobiológicas do TOC (Peng et al., 2012; Whiteside et al., 2004). Dados incongruentes com os achados mais consistentes na literatura da neurobiologia do TOC também foram observados. Foram apresentados resultados indicando volumes diminuídos de substância cinzenta no putâmen, dado na direção oposta ao esperado pela revisão realizada por Peng et al. (2012), na qual encontraram volumes aumentados de substância cinzenta no núcleo lentiforme, que inclui o putâmen. Contrariando o modelo correspondente à hiperatividade no córtex orbitofrontal (Whiteside et al., 2004), o estudo de Freyer et al. (2011) 
apontou para atividade reduzida na área, antes do tratamento, em relação ao grupo controle. Após o tratamento, foi possível observar que a TCC foi capaz de modular neurocircuitos, visto que todos os estudos apresentaram diferenças significativas após as intervenções. A atividade no córtex orbitofrontal foi normalizada em relação ao grupo controle no estudo conduzido por Freyer et al. (2011), e reduzida em Yamanishi et al. (2009). Aumentos da atividade puderam ser observados no córtex cingulado anterior e reduções, no tálamo, correlacionados com a melhora de sintomas (Saxena et al., 2009). Foram encontrados também níveis de ativação menores no córtex pré-frontal (Yamanishi et al., 2009). Em relação ao volume, houve um aumento no córtex cingulado anterior (Hoexter et al., 2011).

Em consonância com o que foi observado, os seguintes estudos recentes apontam para a capacidade de neuromodulação da TCC em diferentes transtornos psiquiátricos. Considerando o Transtorno Depressivo Maior, as evidências indicam que ocorre a normalização da ativação no córtex pré-frontal ventromedial após a TCC (Ritchey et al., 2011), além de indicarem a equivalência em relação à administração de psicofármacos antidepressivos, a partir da atuação de forma diferenciada em mecanismos semelhantes (De Rubeis, Siegle, \& Hollon, 2008). Efeitos na neurocuitaria do medo foram observados nos transtornos de ansiedade (Porto et al., 2014), como no Transtorno de Pânico e Agorafobia (Yang, Kircher \& Straube, 2014), no qual foi notada a normalização da ativação bilateral no giro frontal inferior e mesencéfalo, indicando mudanças no mecanismo de processamento do medo.

Entre os dados coletados pelos estudos após o tratamento, também foi possível observar modulações em dissonância com dados esperados de acordo com a literatura. É o caso de volumes reduzidos encontrados no putâmen e córtex orbitofrontal, que apesar de sofrerem alterações, mantiveramse no padrão compatível com o TOC (Peng et. al., 2012). Já no núcleo caudado, os resultados apontaram para um aumento da atividade e, como o estudo foi realizado a partir de PET, corresponde ao padrão compatível com TOC em curso (Apostolova et al., 2010; Whiteside et al., 2004).

Diante dos resultados, observou-se a importância de modulação de estruturas como córtex orbitofrontal e os núcleos da base no tratamento do TOC. Apesar de não ser considerado um transtorno de ansiedade, o TOC apresenta íntima relação com a categoria (APA, 2013), sendo possível estabelecer um comparativo. Nos transtornos de ansiedade, existe uma interconexão entre o córtex pré-frontal e a amígdala, que são áreas importantes para a integração entre respostas emocionais e cognitivas. A amígdala exerce um papel na aprendizagem e na extinção do medo; seu papel de extinção é exercido juntamente com o córtex préfrontal e o hipocampo. O hipocampo está envolvido nas respostas condicionadas do medo, mas sua ativação pode ser modificada a partir da habituação, que deve levar à extinção do condicionamento. Durante o tratamento, a partir da exposição, o foco da atenção é direcionado à situação ameaçadora, o que faz com que o córtex pré-frontal passe a exercer controle sobre a ativação da amígdala. Assim, projeções inibitórias do hipocampo são criadas nesse novo contexto de informações emocionais, associadas agora a uma situação segura (de Carvalho, Rozenthal, \& Nardi, 2010). No contexto específico do TOC, a região pré-frontal que parece estar mais envolvida é o córtex orbitofrontal, onde foi possível notar uma redução da ativação, que poderia estar relacionada com a redução do esforço para resistir aos pensamentos intrusivos e compulsões. Essa redução do esforço se daria pela extinção do medo, modificando respostas cognitivas e comportamentais (Whiteside et al., 2004; Yamanishiet al., 2009).

Sobre os núcleos da base, é importante destacar que sua modulação representa grande importância sobre o controle de comportamentos indesejados, possibilitando a coordenação e planejamento dos mesmos, conforme explicitado no presente trabalho. Além disso, espera-se que o funcionamento adequado dos núcleos da base e o equilíbrio entre as vias direta e indireta do circuito cortico-estriadotálamo-cortical levem, finalmente, à inibição de impulsos provenientes do tálamo em direção ao córtex, permitindo a redução do foco atencional sobre preocupações e a fixação nos comportamentos repetitivos (Cordioli, 2014; Mercadante et al., 2004). Algumas características dos estudos revisados precisam ser destacadas como limitações passíveis de interferências nos resultados. É o caso de 3 pesquisas que incluíram pacientes com comorbidades em sua amostra (Apostolova et al., 2010; Freyer et al., 2011; Hoexter et al., 2011). Apesar de afetar a homogeneidade da amostra, a inclusão de comorbidades pode ser vista como um meio de estudar o transtorno a partir de um método que conte com amostra mais compatível com sua população, visto que há grande incidência de comorbidades (Hoexter et al., 2011). Pacientes em uso de medicação foram incluídos na amostra de 2 estudos (Yamanishi et al., 2009; Saxena et al., 2009), o que também representa uma limitação, visto que evidências (Hoexter et al., 2011) indicam que psicofármacos envolvidos na inibição da recaptação de serotonina atuam em circuitos neurais diferentes daqueles modulados pela TCC.

Outra limitação metodológica encontrada foi a ausência de grupo controle em dois dos estudos, nos quais a comparação foi entre os momentos anterior e posterior ao tratamento (Apostolova et al., 2010) e entre pacientes responsivos e não responsivos ao tratamento (Yamanishi et al., 2009), o que enfraquece a confiabilidade dos resultados a partir das comparações. A pesquisa realizada por Saxena et al. (2009), apesar de comparar com sujeitos que não apresentavam sintomatologia compatível com TOC, não apresentou os dados relativos ao momento anterior ao tratamento.

Outras limitações observadas tratam das pequenas amostras, da ausência de uniformidade entre o método dos diferentes estudos; do uso de diferentes técnicas adotadas para obtenção de neuroimagem, porém sem replicações na literatura; da seleção de regiões cerebrais pré-estabelecidas para direcionar a investigação. Essas características dificultam a comparação e a generalização dos dados. Dessa forma, verifica-se também a necessidade de que os desenhos experimentais sejam aperfeiçoados e que estudos controlados sobre as diferentes técnicas de neuroimagem sejam replicados. 


\section{Conclusão}

Portanto, observou-se que o TOC tende a se cronificar na ausência de tratamento efetivo, causando diversos prejuízos funcionais. A TCC, a partir da formulação do caso, possibilita o uso de intervenções cognitivas e comportamentais, partindo do alívio dos sintomas em direção à reestruturação de crenças disfuncionais que os mantém. Atualmente, a hipótese mais aceita sobre a neurobiologia envolvida no TOC dá ênfase para uma disfunção no circuito orbitofrontal-subcortical. Os estudos analisados corroboraram a capacidade da TCC na modulação desse neurocircuito. Após o tratamento, foram encontradas alterações no córtex órbitofrontal, núcleo caudado, putâmen, córtex cingulado anterior e córtex pré-frontal. Porém, tendo em vista a discrepância dos recentes achados e as limitações metodológicas apresentadas, o presente estudo aponta na direção da necessidade do desenvolvimento de novas investigações sobre as bases neurobiológicas do TOC, a partir das técnicas de neuroimagem, que podem apontar mudanças cerebais de maneira não invasiva durante tarefas cognitivas e comportamentais, sendo possível identificar as regiões cerebais que mediam a experiência do indivíduo no momento em que elas ocorrem (Gorman, Kent, Sullivan, \& Coplan, 2000), assim como sobre a capacidade de modulação da TCC nos circuitos envolvidos. A partir dos achados, é possível compreender mais claramente os efeitos da TCC no curso do TOC, destacando o papel da EPR e de algumas intervenções cognitivas sobre mecanismos neurais envolvidos no transtorno e consequente redução dos sintomas. Sugere-se, para futuras pesquisas, que as limitações descritas no presente estudo sejam transpostas.

\section{Referências}

American Psychological Association. (2013). DSM-5: Manual diagnóstico e estatístico de transtornos mentais. Porto Alegre, RS: Artmed.

Apostolova, I., Block, S., Buchert, R., Osen, B., Conradi, M., Tabirizan, S., ... Obrocki, J. (2010). Effects of behavioral therapyor pharmacotherapy on brain glucose metabolism in subjects with obsessive-compulsive disorder as assessed by brain FDG PET. Psychiatry Research: Neuroimaging, 184(2), 105-116. doi: 10.1016/j.pscychresns.2010.08.012

Clark, D. A., \& Beck, A.T. (2010a). Cognitive Therapy of Obsessive-Compulsive Disorder. In D. A. Clark \& A.T. Beck (Eds.), Cognitive therapy of anxiety disorders: Science and practice (pp. 446-490). New York: The Guilford Press.

Clark, D. A., \& Beck, A. T. (2010b). Cognitive interventions for anxiety. In D. A. Clark \& A.T. Beck (Eds.) Cognitive therapy of anxiety disorders: Science and practice (pp.180-233). New York: The Guilford Press.

Clark, D. A., \& Beck, A. T. (2010c) Cognitive and Therapy of anxiety and depression: Convergence with neurobiological findings. Trends in Cognitive Sciences, 14(9), 418-424. doi: 10.1016/j.tics.2010.06.007.

Cordioli, A. V. (2008). Vencendo o transtorno obsessivo-compulsivo: Manual de terapia cognitivo-comportamental para pacientes e terapeutas ( $2 \mathrm{a}$ ed.). Porto Alegre, RS: Artmed.
Cordioli, A. V. (2014). Toc: Manual de Terapia Cognitivocomportamental para o transtorno obsessivo-compulsivo (2a ed.). Porto Alegre, RS: Artmed.

Cordioli, A. V., \& Braga, D. T. (2011). Terapia cognitivocomportamental do transtorno obsessivo-compulsivo. In B. Rangé (Org.), Psicoterapias cognitivo-comportamentais: Um diálogo com a psiquiatria (pp.325-345, 2a ed.). Porto Alegre, RS: Artmed.

De Carvalho, M. R., Rozenthal, M., \& Nardi, A. E. (2010). The fear circuitry in panic disorder and its modulation by cognitive-behaviour therapy interventions. The World Journal of Biological Psychiatry, 11(2), 188-198. doi: $10.3109 / 15622970903178176$

Del-Porto, J. A. (2001). Epidemiologia e aspectos transculturais do transtorno obsessivo-compulsivo. Revista Brasileira de Psiquiatria, 23(2), 3-5. Recuperado de http://www.scielo.br/scielo.php?script=sci_arttext\&pid $=$ S1516-44462001000600002

Freyer, T., Klöppel, S., Tüscher, O., Kordon, A., Zurowski, B., Kuelz, A. -K., ..., Voderholzer, U. (2011). Frontostriatal activation in patients with obsessive-compulsive disorder before and after cognitive behavioral therapy. Psychological Medicine, 41(1), 207-216. doi: 10.1017/S0033291710000309

Gomes, P. V. O. (2013). Avaliação da eficácia da inibição da área motora suplementar com estimulação magnética transcraniana de repetição no tratamento do transtorno obsessivo-compulsivo (Unpublished master's thesis), Universidade de Brasília, Faculdade de Ciências da Saúde, Brasília, Brasil. Recuperado de http://repositorio.unb.br/handle/10482/12631

Goodman, W., Price, L., Rasmussen, S., Mazure, C., Fleischmann, R., Hill, C. ,... Charney, D. (November, 1989). The YaleBrown Obsessive Compulsive Scale I. Development, Use, and Reliability. Archives of General Psychiatry, 46(11), 1006-1011. doi: 10.1001/archpsyc.1989.01810110048007.

Goodwin, R. D., Beesdo-Baum, K., Knappe, S., \& Stein, D.J. (2013). Life course epidemiology of anxiety disorders. In K. C. Koenen, S. Rudenstine, E. Susser, \& S. Galea (Eds.), A life course approach to mental disorders (pp. 97-110). United Kingdom: Oxford University Press.

Gorman, J., Kent, J., Sullivan, G., \& Coplan, J. (2000). Neuroanatomical hypothesis of panic disorder, revised. American Journal of Psychiatry, 157(4), 493-505. Recuperado de http:/ovidsp.ovid.com/ovidweb.cgi?T=JS\&PAGE=linkou t\&SEARCH=10739407.ui

Haber, S. N., \& Heilbronner, S. R. (2013). Translational research in OCD: Circuitry and mechanisms. Neuropsychopharmacology, 38(1), 253-253. doi: 10.1038/npp.2012.182

Hoexter, M. Q., Duran, F.L. de S., D'Alcante, C.C., Dougherty, D.D., Shavitt, R. G., Lopes, A.C., ... Busatto, G.F. (2011). Gray matter volumes in obsessive compulsive disorder before and after Fluoxetine or cognitive behavior therapy: A randomized clinical trial. Neuropsychopharmachology, 37(3), 734-745. doi: 10.1038/npp.2011.250

Kircher, T., Arolt, V., Jansen, A., Pyka, M., Reinhardt, I., Kellermann, T., \& Straube, B. (2013). Effect of cognitivebehavioral therapy on neural correlates of fear conditioning in panic disorder. Biological Psychiatry, 73(1), 93-101. doi: 10.1016/j.biopsych.2012.07.026 
Lacerda, A. L. T.de, Dalgalarrondo, P., \& Camargo, E. E. (2001). Achados de neuroimagem no transtorno obsessivo-compulsivo. Revista Brasileira de Psiquiatria, 23(1), 24-27. doi:10.1590/ S1516-44462001000500008

Landeira-Fernandez, J. (2011). Neurobiologia dos transtornos de ansiedade. In B Rangé. (Org.), Psicoterapias cognitivocomportamentais: Um diálogo com a psiquiatria (pp. 68-81, 2a ed.). Porto Alegre, RS: Artmed.

Mercadante, M. T., Rosario-Campos, M. C., Quarantini, L. C., \& Sato, F.P. (2004). As bases neurobiológicas do transtorno obsessivo-compulsivo e da síndrome de Tourette. Jornal de Pediatria, 80(2), 35-44. doi: 10.1590/S002175572004000300006.

Olatunji, B. O., Davis, M. L., Powers, M. B., \& Smits, J. A. J. (2013, January). Cognitive-behavioral therapy for obsessivecompulsive disorder: A meta-analysis of treatment outcome and moderators. Journal of Psychiatric Research, 47(1), 33-41. doi: 10.1016/j.jpsychires.2012.08.020

Peng, Z., Lui, S. S. Y., Cheung, E. F.C., Jin, Z., Miao, G., Jing, J., \& Chan, R. C. K. (2012, December). Brain structural abnormalities in obsessive compulsive disorder: converging evidence from white and grey matter. Asian Journal of Psychiatry, 5(4), 290-296. doi: 10.1016/j.ajp.2012.07.004

Pereira, M., \& Rangé, B. (2011). Terapia Cognitiva. In B. Rangé (Org.), Psicoterapias cognitivo-comportamentais: Um diálogo com a psiquiatria (pp. 20-32, 2a ed.). Porto Alegre, RS: Artmed.
Ritchey, M., Dolcos, F., Eddington, K. M., Strauman, T. J., \& Cabeza, R. (2011). Neural correlates of emotional processing in depression: changes with cognitive behavioral therapy and predictors of treatment response. Journal of Psychiatric Research, 45(5), 577-587. doi: 10.1016/j. jpsychires.2010.09.007

Whiteside, S. P., Port, J. D., \& Abramowitz, J. A. (2004). A metaanalysis of functional neuroimaging in obsessive-compulsive disorder. Psychiatry Research: Neuroimaging, 132(1), 69-79. doi:10.1016/j.pscychresns.2004.07.001

Wright, J. H., Basco, M. R., \& Thase, M. E. (2008). Princípios básicos da terapia cognitivo-comportamental. In J. H. Wright, M. R. Basco, \& M. E. Thase (Eds.), Aprendendo a terapia cognitivo-comportamental: Um guia ilustrado (pp. 15-32). Porto Alegre, RS: Artmed.

Saxena, S., Gorbis, E., O’Neill, J.S., Baker, S.K., Mandelkern, M.A., Maidment, K.M., ... London, D. (2009). Rapid effects of brief intensive cognitive-behavioral therapy on brain glucose metabolism in obsessive compulsive disorder. Molecular Psychiatry, 14(2), 197-205. doi: 10.1038/sj.mp.4002134

Yamanishi, T., Nakaaki, S., Omori, I. M., Hashimoto, N., Shinagawa, Y., Hongo, J., ... Furukawa, T. A. (2009). Changes after behavior therapy among responsive and nonresponsive patients with obsessive-compulsive disorder. Psychiatry Research: Neuroimaging, 172(3), 242-250. doi: 10.1016/j. pscychresns.2008.07.004

Yang, Y., Kircher, T., \& Straube, B. (2014). The neural correlates of cognitive behavioral therapy: Recent progress in the investigation of patients with panic disorder. Behaviour Research and Therapy, 62, 88-96. doi: doi:10.1016/j. brat.2014.07.011 